\title{
El rol de la ingeniería y de la investigación en ingeniería en el proceso de licenciamiento ambiental: una perspectiva desde la sociedad civil $^{1}$
}

\author{
The Role of Engineering and Engineering Science in Environmental Licensing: A Civil Society \\ Perspective
}

\author{
Elsa Matilde Escobar (1), Roberto León Gómez ${ }^{(2)}$ \\ (1) Química Pura de la Universidad de Antioquia. Exjefe de Ciencia y Tecnología del Instituto de Hidrología, Meteorología y Estudios \\ Ambientales (IDEAM). Directora Ejecutiva de la Fundación Natura Colombia. Bogotá, Colombia. elsamescobar@natura.org.co \\ ${ }^{(2)}$ MSc en Economía del Medio Ambiente, Universidad de los Andes. Exdirector de la evaluación de licencias ambientales del Departamento \\ de Planeación Nacional (DNP). Subdirector de Desarrollo Local e Institucional de la Fundación Natura. rlgomez@natura.org.co
}

Recibido 26 de octubre de 2015. Modificado 28 de diciembre de 2015. Aceptado 30 de diciembre de 2015.

DOI: http://dx.doi.org/10.16924/riua.v0i43.903

\begin{abstract}
Palabras clave
Evaluación de impacto ambiental, ética, ingeniería, licencias ambientales, sociedad civil, valor agregado.

\section{Resumen}

En este artículo queremos mostrar la percepción que se tiene desde la sociedad civil del proceso de licenciamiento ambiental en el país. Creemos que es importante pensar la evaluación de impacto ambiental como parte integral de un proceso que da valor agregado a un proyecto. Por esta razón, expondremos algunos principios que consideramos claves en este proceso y hablaremos de los problemas que se presentan. Finalmente, desde una visión ingenieril, propondremos algunas soluciones para lograr que el licenciamiento ambiental sea más efectivo.
\end{abstract}

\section{Key words}

Ethics, Added Value, Civil Society, Environmental Impact Evaluation, Engineering, Environmental Licenses.

\begin{abstract}
In this article, we shed light of civil society's perception of environmental licensing in Colombia. We believe it is important to consider environmental impact assessment as an integral part of a process that adds value to a project. For this reason, we discuss some key principles and discuss the problems that arise. Finally, from an engineering perspective, we propose some solutions to make environmental licensing more effective.
\end{abstract}

\section{INTRODUCCIÓN}

Para empezar contaremos un poco sobre la Fundación Natura. La Fundación es una organización de la sociedad civil creada hace 33 años con el objetivo de velar por la conservación y el uso sostenible de la biodiversidad, por tanto, trabajamos en todas las áreas. Trabajamos en conservación, en herramientas públicas y privadas de conservación, en restauración ecológica, en manejo de cuencas hidrográficas, en cambio climático, en fortalecimiento de las organizaciones locales y de la sociedad civil. A partir de este trabajo que realizamos, creemos que podemos aportar al tema del licenciamiento ambiental dado que somos una organización que trabaja directamente de la mano con las comunidades y con otras organizaciones de la sociedad civil.
Si bien es cierto que nunca hemos hecho un estudio de impacto ambiental, sí hemos apoyado a algunas empresas en la ejecución de sus planes de manejo resultantes de las licencias ambientales. Algunas de las áreas en las que apoyamos esos planes son: monitoreo climático, educación ambiental, restauración ecológica para sus compensaciones, definición de estrategias de compensación, entre

\footnotetext{
1 Una versión preliminar de este artículo fue presentada por Elsa Matilde Escobar y Roberto León Gómez en el foro "El rol de la ingeniería y de la investigación en ingeniería en el proceso de licenciamiento ambiental”, realizado en la Universidad de los Andes el 26 de octubre de 2015.
} 
otros. Esto nos permite tener una interlocución permanente con los ejecutores de las licencias ambientales y con las personas encargadas de las empresas del área ambiental, para brindar nuestro aporte como integrantes y científicos en esta área.

\section{IMPACTO AMBIENTAL VISTO COMO UN PROCESO}

Al hablar de las evaluaciones de impacto ambiental es importante resaltar que son un instrumento básico para cumplir con los conceptos y los lineamientos del desarrollo sostenible. Además, son un medio para que los proyectos se realicen teniendo en cuenta los efectos tanto negativos como positivos ocasionados al medio ambiente. Sin embargo, ni los estudios ni el sistema de impacto ambiental de los proyectos son considerados como un proceso. Cuando nos referimos a proceso, no estamos pensando en las tres etapas del licenciamiento que expuso el viceministro: planeación, evaluación y seguimiento, sino a un trabajo que sea integral al proyecto, a algo que esté presente durante toda la vida útil del proyecto, para que le pueda dar un valor agregado.

Creemos que en el momento en el que el tema del impacto ambiental se convierta en un valor agregado del proyecto $\mathrm{y}$, no sea visto solamente como una obligación, todo será distinto. Esto deben entenderlo tanto los funcionarios del gobierno encargados de otorgar la licencia como los ejecutores de proyectos que las solicitan. Se trata de ver que el tema del impacto ambiental es parte integral de un proyecto porque trae beneficios ambientales y sociales a corto, mediano y largo plazo, y beneficios para las mismas empresas. Además, se convierte en un apoyo a las metas de desarrollo sostenible trazadas por el Gobierno Nacional. Cuando esto se entienda, el proceso de licenciamiento ambiental tendrá otro sentido.

\section{Consideraciones CLAVES}

Para que la evaluación de impacto ambiental pueda ser vista como un proceso, es necesario que cumpla con unas consideraciones claves. Primero, debe tener un propósito. No se trata de hacerla para obtener una licencia o un permiso para iniciar el proyecto. El propósito debe ser proteger el ambiente y lograr el bienestar de la comunidad local y del país en general. Segundo, debe ser un proceso riguroso. En este punto entra la academia. Se debe aplicar la mejor ciencia posible a través del empleo de metodologías y de técnicas apropiadas para señalar esos problemas que se están investigando. Esto no sucede muchas veces. Los estudios suelen pasar por alto los problemas claves a nivel ambiental y social y esto trae inconvenientes tanto para la empresa como para el gobierno.

Tercero, el proceso tiene que ser útil porque debe generar información y productos que ayuden a la resolución de problemas. Estas soluciones tienen que ser aceptables y factibles para que puedan ser llevadas a cabo por los proponentes. Cuarto, se debe proveer información suficiente, confiable y utilizable en la planificación del desarrollo del proyecto y en la toma de decisiones. La información, como se mostró en otras ponencias, debe estar accesible a todo el mundo y debe ser parte fundamental de un sistema. Si bien en Colombia existen muchos sistemas de información, cuando uno los consulta, los documentos presentan hasta dos años de retraso. La información la necesitamos en tiempo real para tomar las decisiones que más convengan al desarrollo sostenible.

En quinto lugar, está el ser costoefectivo. El proceso debe lograr los objetivos del estudio de impacto ambiental a nivel de límites de información, de tiempo, de recursos y de metodologías disponibles. Igualmente, debe ser eficiente en términos de tiempo, de financiamiento para los proponentes y los participantes $\mathrm{y}$, ser congruente para poder cumplir con los requerimientos planteados en el estudio de impacto ambiental. En el sexto lugar, está ser focalizados. El proceso debe concentrarse en los

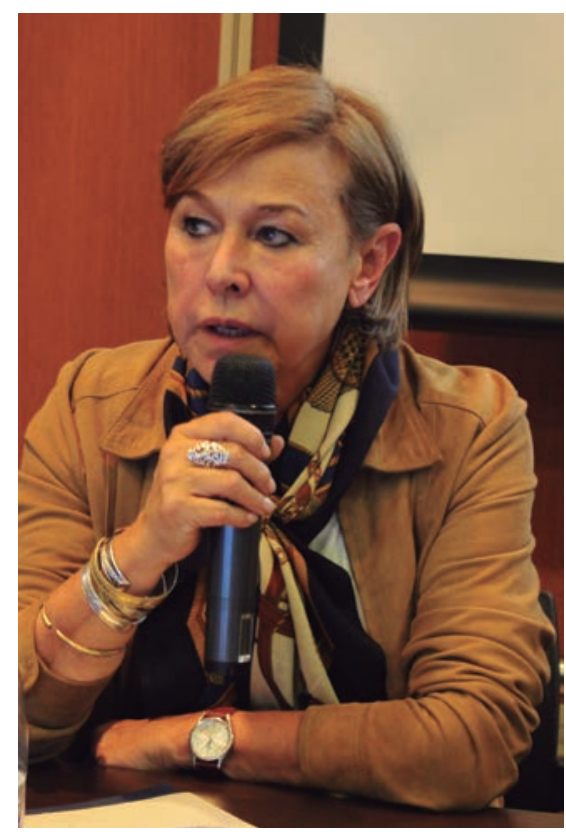

Elsa Matilde Escobar. Fuente: Archivo Particular, Universidad de los Andes

efectos ambientales significativos, en los recursos claves y en los tópicos y temas que son fundamentales para la toma de decisiones. A veces vemos que se solicita una cantidad de información que no es relevante para ninguno de los proyectos y se diluyen los temas importantes para poder proponer las alternativas o las medidas de mitigación de esos impactos que se van a generar.

De igual manera, el proceso debe ser adaptable. Es necesario crear un sistema que se ajuste a las realidades, a los resultados y a las circunstancias de las propuestas revisadas, obviamente, sin comprometer la integridad del proceso mismo. Además, debe ser interactivo incorporando las lecciones aprendidas a partir del ciclo de la propuesta. En muchos casos, se ve que el estudio se hace y resulta ser inmodificable. Nada es inmodificable. En cualquier proceso, no solo en el ambiental, lo más importante es el método adaptativo en el cual uno pueda estar revisando los objetivos, lo que está pasando y la pertinencia de las decisiones tomadas para saber si hay que modificarlas o no.

Es importante también que el proceso sea participativo y provea oportunidades adecuadas para informar 
e involucrar a todo el público interesado y afectado. De esta forma, se podrán recibir sus preocupaciones y señalarlas explícitamente en la documentación para que sean tomadas en cuenta en toma de decisiones. Definitivamente, el involucramiento de la gente es fundamental, sin la gente no tiene sentido hacer proyectos. Los proyectos se hacen por las personas y las afectadas también son ellas.

Además, el proceso tiene que ser interdisciplinario. Si bien la ingeniería tiene un papel importante, hay que hacerles una crítica a los ingenieros. Las firmas de ingenieros a veces no le dan la relevancia a otras disciplinas como la biofísica o las ciencias sociales. Se dedican a copiar estudios hechos en esas áreas con anterioridad y no producen conocimiento nuevo. Quizás esto sucede porque no se contratan a los mejores especialistas o a las personas más capacitadas para hacer los estudios. Quienes están a cargo de esta labor son recién egresados y pasantes y no hay personas que se ocupen de la parte ambiental y social, que son complementarias al área de la ingeniería.

Igualmente, el proceso debe ser verosímil, llevado a cabo con profesionalismo, rigor, honestidad, imparcialidad y equilibrio, y tiene que estar sujeto a comprobaciones y verificaciones independientes. A veces cuando se hacen los estudios de impacto ambiental, queda la duda sobre si los datos presentados son verdaderos o no. Para evitar esta zozobra sería interesante contar con pares externos que hicieran la evaluación y regularan los compromisos posteriores. Otro objetivo es ser transparente. Este tipo de estudios no suelen estar abiertos para que el público los pueda ver y por esta razón, resulta dificilísimo analizarlos y conocerlos en detalle. Uno accede máximo a un resumen. De igual forma, tanto la licencia ambiental como los planes de manejo ambiental y los diagnósticos de alternativas tampoco son puestos a disposición de todos. Finalmente, el proceso debe ser sistémico y considerar en su totalidad toda la información relevante del ambiente afectado, las alternativas propuestas y sus impactos, así como las medidas necesarias para supervisar en investigar los efectos residuales.

\section{DEFICIENCIAS EN LA EVALUACIÓN DE IMPACTO AMBIENTAL}

Vale la pena hacer un análisis crítico de las evaluaciones de impacto ambiental en el país para buscar alternativas que nos lleven a tener un proceso unificado y eficiente. Muchas veces se cambian los criterios de revisión o los requisitos con el respaldo de una sola entidad, sea el Ministerio o el ANLA, y no se consulta a todos los expertos en la materia. Se publican guías o normas sin que haya la participación de los interesados y de la sociedad en general. Aunque el proceso para desarrollar y evaluar los estudios de impacto ambiental esté reglamentado, no unifica criterios ni métodos y difieren sustancialmente entre ellos. No se trata de diferencias en las características biofísicas o culturales de las regiones, sino que depende mucho del funcionario que, de buena fe, decide cambiar los criterios y los métodos. Por ejemplo, hace menos de un mes revisamos dos términos de referencia para unos estudios de impacto ambiental de dos corporaciones colindantes en la misma región. Encontramos que los términos a pesar de no ser completamente distintos, sí diferían muchísimo. Esta es una gran falencia en el sistema que se debe mejorar.

Existen también unos parámetros para determinar si se necesitan o no los estudios de impacto ambiental. Sin embargo, estos parámetros dependen en gran medida de los funcionarios o de las entidades que están a cargo de estos procesos. Ellos deciden la profundidad de los estudios y las exigencias que aplican en cada caso. De esta manera, los términos de referencia y los manuales se reducen a dar sugerencias sobre cómo debería ser llevado a cabo el estudio pero, no existe un método estándar que lleve a la identificación de cada aspecto para que los proponentes de proyectos sepan lo que se les va a evaluar y si los lugares que van a intervenir necesitan de un cuidado especial.

Al conversar con encargados de áreas ambientales, se identificó otra problemática: la falta de personal calificado tanto para evaluar los estudios de impacto ambiental como para hacer el seguimiento. Algunos funcionarios manifiestan que el sistema de estudios de impacto ambiental es gestionado por un número insuficiente de personas que no tienen, en muchos casos, la competencia y el entrenamiento necesarios para hacer la evaluación y el seguimiento requerido. En el caso particular del sector eléctrico, ellos tienen la percepción de que en el ANLA no hay un número adecuado de profesionales con dedicación exclusiva para la evacuación de todos los proyectos en curso. Además, los trámites son vistos como complejos y dispendiosos porque toman mucho tiempo y superan los compromisos contractuales para la realización de los proyectos. Conocemos algunos casos en los que proponentes han desistido de hacer el proyecto porque la tramitología resulta engorrosa y costosa. En nuestro caso, tenemos un proyecto pequeño que cuesta mucho y nos dicen que se demoran seis años en darnos la licencia. Con esto, no hay costos que una organización como la nuestra pueda asumir.

Por otra parte, la legislación colombiana determina la elaboración de un plan de manejo ambiental. Para ello, existe un manual de seguimiento ambiental de proyectos. No obstante, algunas personas que trabajan en el área ambiental nos han comentado también que este manual no se usa de forma adecuada. Es necesario capacitar a las personas encargadas del tema para que el manual sea efectivo. Vale la pena resaltar la iniciativa de crear un sistema de seguimiento ambiental. En ocasiones los funcionarios encargados de hacer las visitas de seguimiento cambian. Esto supone un gran problema porque no hay continuidad en el proceso y se presentan incongruencias. Con la 
creación de este sistema, se unificarían los criterios y el proceso no tendría inconvenientes.

Otro punto que llama la atención es la falta de incentivos para realizar ese licenciamiento ambiental. Como lo decíamos anteriormente, las empresas lo ven como un obstáculo y no como una parte importante que le da valor agregado a su proyecto.

Existe una regulación ambiental y social en el país pero, su aplicación es compleja, genera conflictos, dilata el desarrollo y retrasa la ejecución de los proyectos de infraestructura. Además, hay un conflicto entre la autoridad regional y la autoridad nacional porque no logran ponerse de acuerdo en algunos criterios. Esto se debe a la falta de coordinación y de comunicación entre todos los sectores. Es un hecho que el sistema requiere cambios a nivel metodológico, político, económico y cultural. Sin embargo, creemos que es importante aplaudir las propuestas que se han presentado a lo largo de todas las ponencias de este foro. Esto nos llevará a mejorar y a confiar en el proceso de licenciamiento ambiental del país.

A partir de varios diagnósticos realizados por universidades y académicos que nosotros revisamos sobre la evaluación de impacto ambiental y su implementación, se concluye que el sistema necesita una actualización para mejorar su aplicación. Una metodología estándar podría mejorar notablemente el desarrollo de todo el sistema porque el personal conoce los pasos a seguir y no improvisa de acuerdo a su criterio. Así mismo, la metodología debería ser integral para que pueda verse de manera completa el significado del medio ambiente y la complejidad de los proyectos de desarrollo. Claramente esta metodología tiene que tener en cuenta las características ambientales y sociales de las regiones y el posible impacto de las actividades que se van a realizar en esa región específica, con esa cultura y con ese ambiente determinado. Por esta razón, aunque muchos no estén de acuerdo, consideramos que el análisis de alternativas debe ser obligatorio para todos los proyectos que requieran evaluación de impacto ambiental. De igual manera, los estudios de impacto social deben estar presentes en todos los proyectos y ser complemento de los estudios ambientales. Los criterios deben estar en armonía con las características ambientales y sociales del lugar en el que se realizará el proyecto.

De nuevo hablaremos de la participación pública. Es fundamental que esta participación aumente. En este momento en Colombia la participación está solo para los grupos nativos étnicos pero, la participación ciudadana es indispensable cuando se trata de mejorar la eficiencia y la calidad de los estudios de impacto ambiental. Consideramos que esta consulta debe ser abierta a todos los grupos de la población y las decisiones con respecto a las licencias ambientales tomadas por las autoridades nacionales y regionales deben comunicarse por diferentes medios. Esto con el fin de lograr una protección eficaz del medio ambiente y conservar la diversidad biológica y cultural de Colombia.

Es también fundamental realizar evaluaciones ambientales estratégicas. Aún no se ha reglamentado cuáles proyectos deben tener este tipo de evaluación y cuáles no. Otra debilidad reside en el monitoreo, se podría utilizar el monitoreo comunitario, la misma comunidad es quien mejor conoce su entorno y puede determinar si un proyecto la afecta o no. Al capacitar a la comunidad para que haga el monitoreo se reducen costos y este se hace más efectivo, mucho más que unas visitas esporádicas de unos técnicos o funcionarios de las regiones.

$\mathrm{Si}$ se tienen en cuenta los estudios de impacto ambiental como parte integral del proyecto, se podrían crear unos incentivos para que los proyectos que incluso no tienen que presentar esta evaluación la hicieran y obtuvieran beneficios. En el caso de las licencias exprés, lo importante no es el tiempo sino la calidad de los estudios y de la

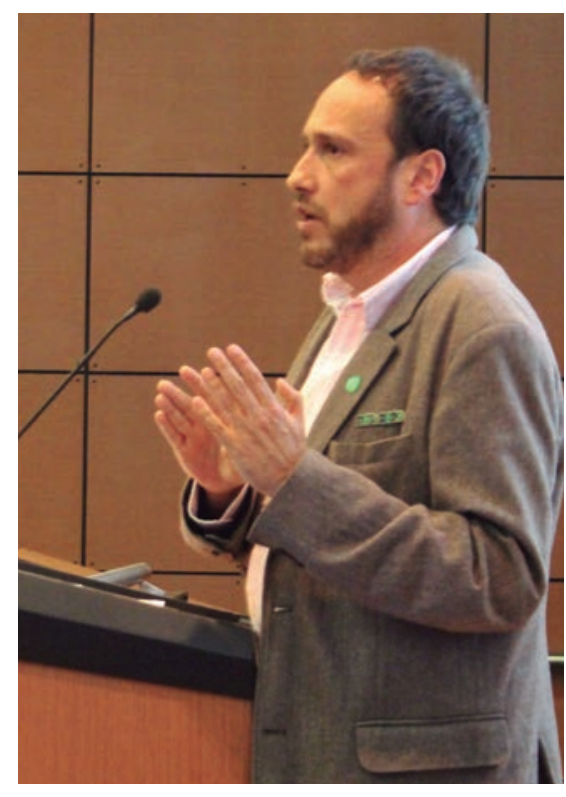

Roberto León Gómez. Fuente: Archivo Particular, Universidad de los Andes

evaluación y contar con el personal necesario para entregar unos resultados positivos al país.

A continuación queremos hablar de algunas acciones que podría emprender la ingeniería para lograr que el proceso de licenciamiento ambiental en el país fuera más efectivo y confiable, que surgen de la experiencia de dirigir la evaluacióndel sistemadelicenciamiento ambiental que se realizó entre 2012 y 2013 para el Departamento Nacional de Planeación. Se hizo un diagnóstico de las tres etapas en las que se divide este proceso. Ahí se encontraron una cantidad de dificultades, aunque también muchos aciertos. Quizás una de las cosas más importantes fue darse cuenta de que los responsables no eran los mismos de siempre. Cuando se habla de las dificultades del proceso de licenciamiento ambiental se mira a la ANLA y a las corporaciones autónomas regionales para buscar en ellos a los responsables de los problemas. No obstante, todos los actores involucrados en este proceso son responsables de lo que sucede.

Para empezar, es necesario mirar cómo se concibe una licencia ambiental. La licencia no es un requisito, es un 
proceso de toma de decisiones y la herramienta de gestión ambiental más importante con la que contamos en el país. El problema está en que los sectores productivos la están asumiendo como un trámite o como un derecho adquirido por estar realizando un proyecto. La Corte Constitucional ha sido clara en decir que la licencia ambiental no es un derecho adquirido. El hecho de desarrollar un proyecto, bien sea porque el gobierno nacional le ha otorgado a uno la concesión o porque la iniciativa privada decide hacer ese proyecto, no implica que la autoridad ambiental tenga que dar la licencia ambiental. La licencia ambiental es un proceso de decisión y de gestión sectorial y territorial.

El proceso de licenciamiento ambiental se debe realizar adecuada y oportunamente. Coincidimos en lo que se ha presentado en otras ponencias sobre el desfase existente entre los diagnósticos ambientales de alternativas, los estudios de impacto ambiental, los análisis de restricciones ambientales y las fases de ingeniería. Por ejemplo, se exige un diseño detallado del proyecto cuando apenas se va a hacer el estudio de impacto ambiental. Este estudio debería realizarse antes, en una fase más temprana, no en el momento de hacer el diseño final del proyecto. En el caso del diagnóstico ambiental de alternativas, este debería ir en la fase de pre factibilidad, donde se están analizando las alternativas del proyecto. Este problema no pueden solucionarlo los ingenieros que están del lado de la consultoría, sino aquellos que están en la autoridad ambiental.

En cuanto a la falta de información, es necesario trabajar conjuntamente con los distintos actores para generar información con escalas suficientes para la toma de decisiones. Las escalas que tenemos en este momento no nos permiten tomar decisiones a nivel de proyectos y la responsabilidad de generar la información queda en manos de profesionales, proponentes de proyectos o consultores. Ahí hay ingenieros, geólogos, geomorfólogos, hidrólogos, climatólogos, biólogos, ingenieros forestales, economistas, sociólogos, antropólogos, entre otros. Esto lo que muestra es la importancia del trabajo interdisciplinario al que los ingenieros no estamos acostumbrados. Los ingenieros somos arrogantes y creemos que nuestro trabajo es el más importante. Eso no es así. Tenemos que aprender a trabajar y a dialogar con las otras disciplinas, a aprender cómo piensan desde otras áreas del conocimiento. Es difícil, pero se puede empezar desde la universidad cuando los profesionales están en formación.

El tema del personal, del recurso humano en el proceso de licenciamiento ambiental es clave. Tal como lo proponía Ángela Cadena, por sugerencia de Marcela Bonilla, es muy importante que desde la academia se trabaje interdisciplinariamente. Por ejemplo, en la carrera de ingeniería civil, en la clase de diseño geométrico de vías, se le pedía a los estudiantes que trazaran una vía teniendo en cuenta una topografía y unos ríos que cruzaban esa topografía. El estudiante traza la vía pensando en los criterios que le han enseñado: compensar llenos y cortes para que la vía salga más barata. Nunca le enseñan al estudiante que debe pensar si hay comunidades étnicas en ese trazado, o un área protegida o algún humedal. De ahí que los ingenieros caigamos en el error de creer que lo único importante es la vía o el puente o la línea de transmisión. Estamos dejando de lado aspectos fundamentales para lograr que el desarrollo sea sostenible. No se puede hacer una vía si en la mitad del terreno hay un área protegida.

Un caso de la vida real es el retraso de la vía del Sol, producido porque los concesionarios "de repente" descubrieron que existía la reserva forestal de San Francisco. Ni el Instituto de Vías, ni la Agencia Nacional de Infraestructura, ni nadie lo había descubierto. Vino a descubrir esto el concesionario, obviamente una vez se ganó la licitación. Como pueden ver, esto indica claramente que tenemos un problema muy serio porque no estamos considerando los temas ambientales en el diseño de nuestros proyectos. Esto hace que se ponga en juego la credibilidad de disciplinas como la ingeniería. Con los escándalos de los últimos años, tanto del edificio Space en Medellín, como de los contratos con los Nule en Bogotá, por citar solo algunos ejemplos, se está dañando la reputación de los ingenieros.

$\mathrm{Y}$ aquí hay un tema que nadie ha tocado: la ética profesional. Esto es clave en una profesión como la ingeniería y más, cuando se trabaja en temas de licenciamiento ambiental. La ética, aunque en la universidad dicten cursos, es un tema personal, de formación familiar que recibimos todos y de la forma como nos comportamos como seres sociales. Lo voy a ilustrar con el caso de la construcción de una línea de transmisión; la ANLA se demora en el proceso más de los 90 días reglamentarios y se toma 180 o 200 días y los usuarios piensan que es el colmo que la autoridad ambiental retrase tanto los procesos. Sin embargo, no se muestra algo perverso que sucede en muchas ocasiones: Los consultores presentan estudios de impacto ambiental incompletos o de baja calidad y obligan a la autoridad ambiental a solicitar nueva información. Con todo y eso, en la segunda entrega de información a veces siguen entregando documentación incompleta apostándole un poco a sacar rápido la licencia. Es claro que no se puede generalizar pero, a veces sucede.

Cuando hicimos la evaluación del sistema ambiental del país nos dimos cuenta que ese ir y venir entre la autoridad ambiental y el peticionario es constante, lo que indica que hay unos estándares éticos muy bajos en algunos consultores y esa es la razón por la que presentan estudios incompletos. La autoridad ambiental tiene que valerse de cualquier cantidad de instrumentos para poder obtener la mejor información y así tomar la decisión más adecuada.

En cuanto a la consulta previa y la participación social, hay que decirlo claramente, son vistas como un obstáculo. Por ejemplo, los desarrolladores 
de proyectos piensan que mientras el Ministerio del Interior les busca y les entrega el mapa del territorio étnico se generan demoras. Aquí es necesario aclarar que hay grupos étnicos con territorio y sin territorio $\mathrm{y}$, en ambos casos, se debe hacer consulta. No hay nada que hacer. Estas variables hay que tenerlas en cuenta cuando se va a llevar a cabo una obra. Se debe tener claro que la participación de estos grupos y comunidades es clave porque las obras de ingeniería dan solución a problemas reales de la gente. Lo importante es brindar una solución, no la obra en sí. Si el proyecto tiene como fin solucionar un problema de la gente, es indispensable oírlos, conocer sus inquietudes y resolverles las dudas con respecto a la obra. A veces por solucionarles el problema a unos, se afecta a otros. Eso hay que mirarlo. Es un tema que le da valor agregado al proceso de licenciamiento porque la comunidad se convierte en una fuente de información más.

Queremos terminar recordando que la palabra ingeniero tiene una relación muy estrecha con la palabra ingenio. Ingenioso es aquel capaz de proveer soluciones innovadoras y distintas a los problemas. No se les puede olvidar eso. No podemos convertirnos en ingenieros que repiten fórmulas que están en los libros. Debemos buscar soluciones diferentes e innovadoras a los problemas de la comunidad. Eso hace parte del desarrollo de una buena carrera profesional.

\section{REFERENCIAS}

Fundación Natura y S.E.I. Consultores. (2013). Evaluación de operaciones al proceso de licenciamiento ambiental en sus etapas de planeación, evaluación y seguimiento, desarrollados por las entidades encargadas del sector, los peticionarios y las autoridades ambientales - Informe Final. Bogotá: Departamento Nacional de Planeación. 